REVISTA INTERNACIONAL DE CIENCIAS DEL DEPORTE International Journal of Sport Science

Rev. int. cienc. deporte

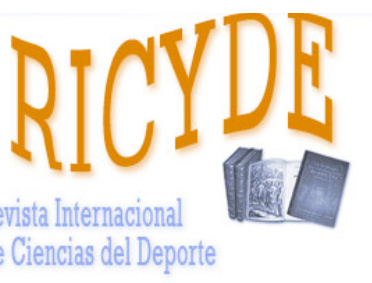

International Journal of Sport Science

VOLUMEN IX - AÑO IX

Páginas:114-125 ISSN:1885-3137

No 32 - Abril - 2013

http://dx.doi.org/10.5232/ricyde2013.03202

\title{
Indicadores de rendimiento ofensivo en el floorball de alto nivel Offensive performance indicators of high level floorball
}

\author{
Miguel Prieto-Gómez \\ Seleccionador del equipo masculino absoluto de España de Floorbal \\ Javier Pérez-Tejero y Miguel Ángel Gómez \\ Universidad Politécnica de Madrid
}

\section{Resumen}

El objetivo del presente estudio fue obtener información específica sobre los indicadores de rendimiento ofensivos más relevantes en competición en floorball de alto nivel. Para ello, fueron analizadas las acciones ofensivas (AO) que acaban en lanzamiento de 14 partidos internacionales de las temporadas 2008 a $2010(n=1500)$. Se utilizó un diseño de investigación descriptivo y de tipo correlacional, a partir de la aplicación de un instrumento de observación específico para las AO distinguiendo según el origen, desarrollo, finalización y resultado de cada acción ofensiva. Para analizar el grado de asociación entre las variable analizadas se utilizaron tablas de contingencia (Chi-cuadrado de Pearson - $x^{2}-y$ el coeficiente de contingencia) en relación al éxito o no éxito final de cada $A O$, estableciéndose el nivel de confianza en $\mathrm{p} \leq 0,05$. Los resultados muestran que el floorball es un deporte de $A O$ de corta duración (el $69 \%$ duraron menos de 15 segundos: $8,9 \pm 0,9)$, donde el contraataque y la técnica de lanzamiento mediante la elevación de la pala por debajo de la rodilla son las variables que permiten obtener más éxito $(p<0,001)$. El contraataque fue menos frecuente que el ataque posicional $(31,3 \% \mathrm{vs} .68,7 \%$, $\mathrm{p}<0,001)$. La actuación del portero es determinante, así como la finalización de las AO en la zona más próxima a portería.

Palabras clave: floorball; acciones ofensivas; deportes de equipo; metodología observacional; análisis del juego.

\section{Abstract}

The aim of the present study was to obtain specific information about the offensive performance indicators in high level floorball competitions. The analysis of offensive actions (OA) in high-level men's competition release was used. The sample included 14 International games of the 2008-2010 seasons $(n=1500)$. A design of descriptive and correlational research was used, from the application of a specific observation instrument for OA analysis distinguishing between starting, development, completion and final of a given OA. To analyze the degree of association between variables the crosstabs command was used (Pearson's chi-square $-x^{2-}$, and the coefficient of contingency) in relation to the effectiveness of each $O A$. The significant level was set at $p \leq 0,05$. The results showed that floorball was characterized by short possession durations (69\% lasted less than 15 seconds: $8,9 \pm 0,9$ ), where counterattacks, and the shooting technique of elevation of the blade below the knee are the variables that were more related to OA effectiveness $(p<0,001)$. Counterattack was less frequent than positional offense $(31,3 \%$ vs. $68,7 \%, p<0,001)$. The performance of goalkeeper was relevant as well as the completion of the OA in the area closer to the goal.

Key words: floorball; offensive actions; team sports; observational methodology; notational analysis.

Correspondencia/correspondence: Miguel Prieto Gómez

Facultad de Ciencias de la Actividad Física y del Deporte -INEF. Universidad Politécnica de Madrid.

E-mail: mprieto@unihockey.es 


\section{Introducción}

$\mathrm{E}$ análisis del rendimiento en deportes de equipo ha puesto de manifiesto la importancia del estudio del rendimiento individual y de equipo en diferentes contextos de juego, con el objeto último de obtener información para la mejora de las situaciones de entrenamiento en relación a las demandas competitivas (Hughes y Bartlett, 2002; Sampaio, Lago, y Drinkwater, 2010). De hecho, el rendimiento en deportes de equipo se ve afectado por variables como la calidad del oponente, el periodo dado de juego, la localización del partido (casa / fuera), el estatus del juego y el tipo de competición (Gómez y Pollard, 2011; Lago, 2009; Lago y Martín, 2007; Sampaio, Lago, Casais, y Leite, 2010). Estos efectos e interacciones han sido estudiados de manera extensa en la bibliografía de diferentes deportes de equipo. Según la categorización de Hughes y Bartlett (2002), el floorball se puede incluir dentro de los deportes colectivos de oposición con un espacio común. Aunque incluido en esta clasificación, no se encuentra en la literatura especializada un estudio que analice los indicadores de rendimiento, tal y como ha ocurrido en otros deportes de equipo de la familia del hockey, como el hockey patines (Kingman y Dyson, 2001; Yagüe, 2005), el hockey hierba (Gabbett, 2010; Piñeiro, 2008) o el hockey hielo (Geithner, Lee, y Bracko, 2006), o en otros deportes de referencia en el floorball como el baloncesto (Gómez y Lorenzo 2005; Manzano, Lorenzo, y Pacheco, 2005; Gómez, Lorenzo, Ortega, y Olmedilla, 2007; Pollard y Gómez, 2007; Sampaio, Ibáñez, Gómez, Lorenzo, y Ortega, 2008; Ortega, Fernández, Ubal, Lorenzo, y Sampaio, 2010).

Los sistemas notacionales informatizados aplicados a los deportes de oposición han permitido analizar y cuantificar los indicadores de rendimiento, facilitando la labor del entrenador, la evaluación táctica y la efectividad de las acciones de juego (Hughes y Bartlett, 2002). Sin embargo, estas técnicas de observación, tanto para el análisis de los gestos técnicos desde un punto de vista biomecánico, como para el análisis de la acción de juego a través de la observación indirecta, aún no han sido implementadas en el floorball (Prieto y Pérez, 2011), a pesar de que se han mostrado muy eficaces en otros deportes colectivos. En relación al floorball solo se ha encontrado la referencia anterior en relación al análisis del rendimiento en la liga sueca, en el que se analizaron tres partidos del play-off de la liga 2005-2006, destacando la importancia del contraataque dentro de los sistemas de juego utilizados por los equipos (supone el $25 \%$ de los ataques y obtiene el $50 \%$ de los goles), la relación significativa entre la zona de finalización y el resultado final de la acción, y la relevante participación del portero en el resultado de las acciones.

Por todo lo anterior, las limitaciones presentadas a nivel de investigaciones sobre el análisis del juego en el floorball, sugieren la necesidad de un análisis acerca del conocimiento de cómo se desarrollan las acciones ofensivas y los lanzamientos, ya que son uno de los factores determinantes de este deporte, para poder adecuar los entrenamientos a la exigencia competitiva real y poder incidir sobre los indicadores de rendimiento específicos del deporte (Vales y Areces, 2009). En esta línea, se hace necesario desarrollar estudios que utilicen metodologías observacionales que permitan implementar y mejorar el rendimiento en entrenamientos y en competición (Anguera, 2009). Por ello, el objetivo del presente estudio fue analizar las acciones ofensivas que terminan en lanzamiento en el floorball de alto nivel, tratando de obtener información sobre qué variables permiten obtener más éxito en la finalización de los ataques. 


\section{Material y método}

\section{Muestra}

Se han analizado 1500 acciones ofensivas (AO) pertenecientes a 14 partidos internacionales masculinos entre los años 2008 y 2010 del Torneo Internacional Cuatro Naciones y las fases de clasificación para Campeonatos del Mundo.

\section{Procedimiento}

Los 14 partidos se analizaron por dos observadores expertos mediante observación indirecta aplicando una metodología observacional diseñada ad hoc. Los observadores fueron 2 licenciados en Ciencias de la Actividad Física y del Deporte, con más de 5 años de experiencia como entrenadores de floorball, asimismo se realizaron los procedimientos de formación de observadores propios de este tipo de estudios (Anguera, 2009). Para comprobar la fiabilidad de las observaciones se realizaron análisis posteriores de observación de 2 partidos completos seleccionados de manera aleatoria, mostrando el índice Kappa valores superiores a 0,81 (Altman, 1991).

\section{Variables}

Las variables de estudio fueron definidas siguiendo a Prieto y Pérez (2010) en base a las características específicas de la AO, estableciendo las siguientes: tipo de ataque, duración de la $\mathrm{AO}$, número de jugadores que participan en el ataque, número de pases por ataque, la altura del último tiro, la trayectoria del último pase, la clase de recuperación, el grado de oposición defensivo al tiro, así como la zona de origen y finalización de la $\mathrm{AO}$, utilizándose una división del campo de juego validada por criterio experto a través de cuestionario (Prieto y Pérez, 2009; figura 1). Estas variables y la propia división del campo fueron validadas por criterio experto a través de un cuestionario realizado al efecto y administrado a entrenadores internacionales de floorball $(\mathrm{n}=8)$, que alcanzaron los tres siguientes criterios de inclusión para su participación en aquel estudio: a) estar entrenando en la actualidad (momento del estudio) y acumular al menos 5 años de experiencia; b) el trabajo profesional se podía desempeñar o haberse desempeñado en el equipo técnico de selecciones nacionales (mínimo un año) o entrenando a un equipo de élite de liga nacional (máxima categoría de cada país) al menos un año o tener más de 10 años de experiencia en equipos de categorías inferiores, y c) estar en posesión de al menos el título de entrenador nivel II reconocido por la International Floorball Federation (Prieto y Pérez, 2009).
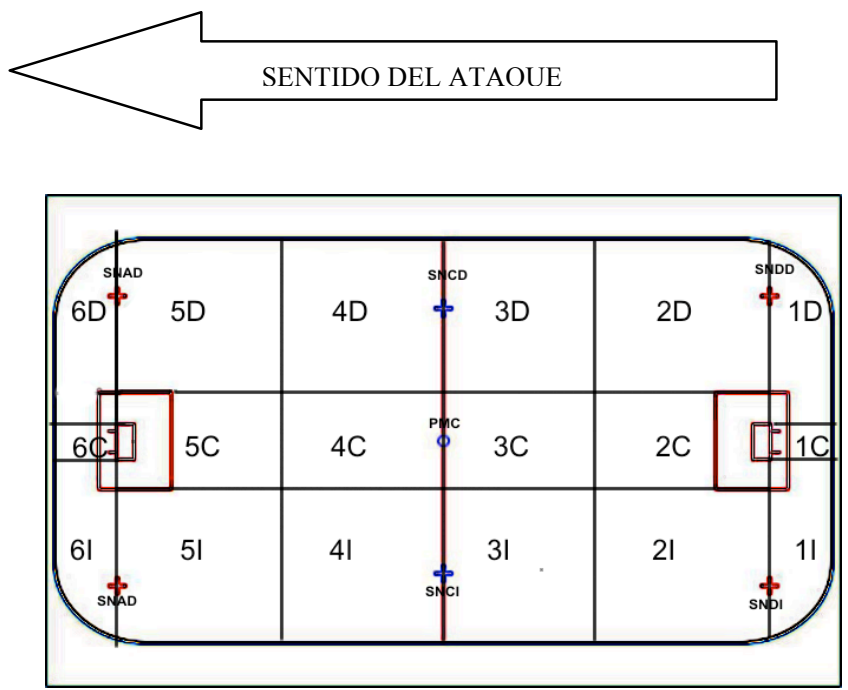

Figura 1. División del campo de juego en zonas (Prieto y Pérez 2009). 


\section{Análisis estadístico}

Para estudiar la muestra se ha realizado un análisis por variables calculando los estadísticos descriptivos de cada una de ellas (media y desviación típica). El tipo de dato de este estudio, salvo la duración de la AO, son datos de tipo intervalo y categorías. Además, las observaciones son independientes y las categorías diseñadas mutuamente excluyentes. Por todo ello se decidió utilizar estadística no paramétrica (Thomas y Nelson, 2007). El análisis correlacional se ha efectuado a través de la construcción de tablas de contingencia y los valores estadísticos del $\chi^{2}$ de Pearson y el coeficiente de contingencia. Por otro lado, dentro de las frecuencias de cada variable, se destaca la importancia del valor de los residuos corregidos tipificados. Los datos fueron tratados con el paquete estadístico SPSS para Windows, v.18.0 (Chicago, IL). El nivel de significación se estableció en $\mathrm{p} \leq 0,05$.

\section{Resultados}

La media de AO por partido es de $107,1( \pm 18,7)$, de las cuales un $38,5 \%$ acaban en éxito y un $61,5 \%$ son acciones falladas (26\%) o tapadas por un jugador de campo $(35,5 \%)$. El 7,4\% de las AO totales termina en gol, pero si consideramos solamente las AO que terminan en éxito, (las AO que terminan llegando a portería o a situación de penalti), el gol representa poco menos de un $20 \%$. El portero interviene, por tanto, en el $80 \%$ de las $\mathrm{AO}$ estudiadas que acaban entre los tres palos (tabla 1).

Tabla 1. Porcentajes del resultado final de la acción ofensiva.

\begin{tabular}{|c|c|c|c|}
\hline \multicolumn{2}{|c|}{ RESULTADO } & Frecuencia & Porcentaje \\
\hline \multirow{4}{*}{ ÉXITO } & Gol & 111 & 7,4 \\
\cline { 2 - 4 } & Parada Portero con Posesión & 152 & 10,1 \\
\cline { 2 - 4 } & Rechace del Portero & 292 & 19,5 \\
\cline { 2 - 4 } & Tiro al Poste o Larguero & 4 &, 3 \\
\cline { 2 - 4 } & Situación Penalti & 18 & 1,2 \\
\hline \multirow{2}{*}{ FALLO } & Tiro Fallado & 390 & 26,0 \\
\cline { 2 - 4 } & Tiro Tapado por Jugador de Campo & 533 & 35,5 \\
\hline & Total & 1500 & 100 \\
\hline
\end{tabular}

El contraataque es menos frecuente que el ataque posicional (31,3\% vs $68,7 \%)$. Con el contraataque se obtiene un $48,4 \%$ de éxito por un $34 \%$ del ataque posicional. Los valores del $\chi^{2}$ de Pearson $(\mathrm{p}<0,001)$ de la tabla de contingencia que incluye las variables "Tipo de ataque" y "Éxito o fallo" indican que no hay independencia entre esas variables. Los residuos tipificados corregidos positivos del contraataque con respecto al éxito $(5,3)$ indican que ambos están significativamente relacionados (tabla 2). 
Tabla 2. Tabla de contingencia que relaciona el "Tipo de ataque" y el "Éxito o fallo".

\begin{tabular}{|c|c|c|c|c|}
\hline \multirow{2}{*}{\multicolumn{2}{|c|}{ TIPO DE ATAQUE }} & \multicolumn{2}{|c|}{ ÉXITO / FALLO } & \multirow{2}{*}{ Total } \\
\hline & & Éxito & Fallo & \\
\hline \multirow{2}{*}{ Ataque Posicional } & Recuento & 351 & 680 & 1031 \\
\hline & Residuos corregidos & $-5,3$ & 5,3 & \\
\hline \multirow[t]{2}{*}{ Contraataque } & Recuento & 227 & 242 & 469 \\
\hline & Residuos corregidos & 5,3 & $-5,3$ & \\
\hline Total & Recuento & 578 & 922 & 1500 \\
\hline
\end{tabular}

La mayoría de las AO (69\%) se producen en menos de 15 segundos, siendo ocasionales aquellas que duran más de 30 segundos. La duración media de la AO fue de $8,9( \pm 0,9)$ segundos, consiguiéndose el 55\% de los goles en el rango de 0-10 segundos. La media de jugadores implicados es de $2,8( \pm 1,3)$ y la media de pases por AO fue de $3,1( \pm 1,2)$. Cerca del $84 \%$ de las AO se realizan en situaciones de igualdad numérica de 5 contra 5. La altura del último pase mayoritariamente usada es la rasa (88\%), y dos técnicas (la de arrastre y la de elevación de la pala por debajo de la rodilla) representan el $74 \%$ del total de las técnicas utilizadas para el lanzamiento. Esta última técnica se muestra como la más eficaz en su relación con los resultados finales de la acción ofensiva $\left(\chi^{2} \mathrm{p}<0,001\right.$, coeficiente de contingencia 0,23 ), en concreto con la más determinante, el gol, ya que se obtiene el $62 \%$ del total con solo el $41 \%$ de las AO que representa esta técnica, además de obtener menos tiros tapados de los esperados estadísticamente.

La trayectoria del último pase más habitual es diagonal adelante $(40,2 \%)$, seguida por la diagonal atrás $(25,3 \%)$. La altura del lanzamiento preponderante es la media $(57,5 \%)$ seguida por la alta (31\%) que consigue casi el $46 \%$ de los goles. En cuanto al origen de la AO destaca que la recuperación directa es mucho más frecuente que la indirecta $(64,3 \%$ por $35,7 \%)$. Los residuos de la tabla 3 nos indican además que la recuperación directa tiene residuos corregidos tipificados significativos con respecto al éxito $(+4,6)$, siendo lo contrario en el caso de la recuperación indirecta $(-4,6)$.

Tabla 3. Tabla de contingencia según "Clase de recuperación” y "Éxito o fallo".

\begin{tabular}{|c|c|c|c|c|}
\hline \multirow{2}{*}{ CLASE RECUPERACIÓN } & \multicolumn{2}{c|}{ ÉXITO / FALLO } & \multirow{2}{*}{ Total } \\
\cline { 2 - 5 } & Recuento & Éxito & Fallo & \multirow{2}{*}{965} \\
\hline \multirow{2}{*}{ Directa } & Residuos corregidos & 4,6 & 552 & 535 \\
\cline { 2 - 5 } & Recuento & 165 & 370 & \\
\hline \multirow{2}{*}{ Indirecta } & Residuos corregidos & $-4,6$ & 4,6 & 1500 \\
\hline
\end{tabular}


Teniendo en cuenta el lugar de finalización, la zona 5C (la zona más próxima y frontal a portería, figura 1) obtiene resultados positivos en todas las situaciones de éxito (tabla 4). Sin duda, esta zona sobresale por sus residuos tipificados corregidos significativos en varias de las variables de "Resultado" (ver tabla 3 ): el gol $(7,5)$, el rechace del portero $(4,6)$ y el tiro al poste o larguero $(2,1)$ en el apartado positivo y, el tiro fallado $(-4,0)$ y tiro tapado $(-4,8)$ que son finalizaciones de no éxito, obtienen en esta zona menos valores de los esperados estadísticamente. Sin embargo, las zonas 4I, 4D y 4C (ver figura 1) obtienen resultados negativos con la variable gol $(-3,2,-2,3$ y $-2,6$ respectivamente) y mayor número de $\mathrm{AO}$ de tiro tapado $(2,5$ para $4 \mathrm{I}$ y $4 \mathrm{D}$ y 5,0 para $4 \mathrm{C})$. Además, desde $4 \mathrm{D}$ y $4 \mathrm{C}$ se consiguen menos rechaces del portero de los esperados $(-2,0$ y $-3,0)$. Con todo, el $\chi^{2}$ y el índice del coeficiente de contingencia $(0,36)$ son significativos $(p<0,001)$, con un grado de asociación medio. Todas las zonas citadas pueden consultarse en la tabla 4.

Tabla 4. Frecuencia y residuos corregido de Resultados de la acción ofensiva en las zonas 5C,4C, 4I y 4D.

\begin{tabular}{|c|c|c|c|c|c|c|c|c|c|}
\hline \multirow{2}{*}{\multicolumn{2}{|c|}{$\begin{array}{c}\text { ZONA } \\
\text { FINALIZACIÓN }\end{array}$}} & \multicolumn{7}{|c|}{ RESULTADO } & \multirow{3}{*}{$\begin{array}{r}\text { Total } \\
350\end{array}$} \\
\hline & & \multirow{2}{*}{$\begin{array}{c}\text { Gol } \\
58\end{array}$} & \multirow{2}{*}{$\begin{array}{c}\begin{array}{c}\text { Parada } \\
\text { Portero } \\
\text { con } \\
\text { Posesión }\end{array} \\
35\end{array}$} & \multirow{2}{*}{$\begin{array}{c}\begin{array}{c}\text { Rechace } \\
\text { del } \\
\text { Portero }\end{array} \\
98\end{array}$} & \multirow{2}{*}{$\begin{array}{c}\begin{array}{c}\text { Situación } \\
\text { Penalti }\end{array} \\
2\end{array}$} & \multirow{2}{*}{$\begin{array}{c}\text { Tiro al } \\
\text { Poste o } \\
\text { Larguero }\end{array}$} & \multirow{2}{*}{$\begin{array}{c}\text { Tiro } \\
\text { Fallado } \\
62\end{array}$} & \multirow{2}{*}{$\begin{array}{c}\text { Tiro } \\
\text { Tapado } \\
87\end{array}$} & \\
\hline \multirow{2}{*}{$5 \mathrm{C}$} & Recuento & & & & & & & & \\
\hline & $\begin{array}{l}\text { Residuos } \\
\text { corregidos }\end{array}$ & 7,5 &,- 1 & 4,6 & 1,3 & 2,1 & 4,0 & $-4,8$ & \\
\hline \multirow{2}{*}{$4 \mathrm{C}$} & Recuento & 5 & 14 & 21 & 0 & 1 & 49 & 97 & 187 \\
\hline & $\begin{array}{l}\text { Residuos } \\
\text { corregidos }\end{array}$ & $-2,6$ & $-1,3$ & $-3,0$ &,- 8 &,- 9 &, 1 & 5,0 & \\
\hline \multirow{2}{*}{$4 \mathrm{D}$} & Recuento & 5 & 21 & 23 & 0 & 0 & 44 & 74 & 167 \\
\hline & $\begin{array}{l}\text { Residuos } \\
\text { corregidos }\end{array}$ & $-2,3$ & 1,1 & $-2,0$ &,- 7 & $-1,5$ & ,1 & 2,5 & \\
\hline \multirow{2}{*}{$4 \mathrm{I}$} & Recuento & 2 & 23 & 24 & 1 & 0 & 40 & 72 & 162 \\
\hline & $\begin{array}{l}\text { Residuos } \\
\text { corregidos }\end{array}$ & $-3,2$ & 1,8 & $-1,6$ & ,9 & $-1,5$ &,- 4 & 2,5 & \\
\hline
\end{tabular}


Tabla 5. Tabla de contingencia relacionando la variable Grado de oposición defensiva al tiro (GOTI) y el "Éxito / fallo".

\begin{tabular}{|c|c|c|c|c|}
\hline \multicolumn{2}{|c|}{ GRADO DE OPOSICIÓN DEFENSIVA AL TIRO } & \multicolumn{2}{|c|}{ ÉXITO / FALLO } & \multirow{3}{*}{$\begin{array}{c}\text { Total } \\
225\end{array}$} \\
\hline & & \multirow{2}{*}{$\begin{array}{c}\text { Éxito } \\
68\end{array}$} & \multirow{2}{*}{$\begin{array}{c}\text { Fallo } \\
157\end{array}$} & \\
\hline \multirow{4}{*}{ Alto } & Recuento & & & \\
\hline & $\%$ de GOTI & $30,2 \%$ & $69,8 \%$ & $100,0 \%$ \\
\hline & \% de Éxito/Fallo & $11,8 \%$ & $17,0 \%$ & $15,0 \%$ \\
\hline & Residuos corregidos & $-2,8$ & 2,8 & \\
\hline \multirow{4}{*}{ Medio } & Recuento & 141 & 306 & 447 \\
\hline & $\%$ de GOTI & $31,5 \%$ & $68,5 \%$ & $100,0 \%$ \\
\hline & \% de Éxito/Fallo & $24,4 \%$ & $33,2 \%$ & $29,8 \%$ \\
\hline & Residuos corregidos & $-3,6$ & 3,6 & \\
\hline \multirow{4}{*}{ Bajo } & Recuento & 353 & 454 & 807 \\
\hline & $\%$ de GOTI & $43,7 \%$ & $56,3 \%$ & $100,0 \%$ \\
\hline & \% de Éxito/Fallo & $61,1 \%$ & $49,2 \%$ & $53,8 \%$ \\
\hline & Residuos corregidos & 4,5 & $-4,5$ & \\
\hline \multirow{4}{*}{ Nulo } & Recuento & 16 & 5 & 21 \\
\hline & $\%$ de GOTI & $76,2 \%$ & $23,8 \%$ & $100,0 \%$ \\
\hline & \% de Éxito/Fallo & $2,8 \%$ &, $5 \%$ & $1,4 \%$ \\
\hline & Residuos corregidos & 3,6 & $-3,6$ & \\
\hline \multirow{3}{*}{ Total } & Recuento & 578 & 922 & 1500 \\
\hline & $\%$ de GOTI & $38,5 \%$ & $61,5 \%$ & $100,0 \%$ \\
\hline & \% de Éxito/Fallo & $100,0 \%$ & $100,0 \%$ & $100,0 \%$ \\
\hline
\end{tabular}

Cuando el grado de oposición al tiro (GOTI) es alto, el porcentaje de éxito baja considerablemente (tabla 5), y cuando éste es bajo o nulo, las situaciones de resultado de éxito, especialmente el gol, aumentan considerablemente. En este sentido, el GOTI desciende si se produce el lanzamiento sin control previo, tal y como indica la tabla 6, ya que el GOTI alto obtiene residuos tipificados negativos $(-2,8)$ con respecto al tiro directo y el GOTI bajo tiende a relacionarse con el aumento del uso del control previo al tiro $(1,7 ; p=0,032)$, existiendo efectivamente diferencias significativas. 
Tabla 6. Tabla de contingencia que relaciona el "Grado de oposición defensiva al tiro" (GOTI) y "Control previo al tiro".

\begin{tabular}{|c|c|c|c|c|}
\hline \multirow{2}{*}{ GRADO DE OPOSICIÓN DEFENSIVA AL TIRO } & \multicolumn{2}{c|}{ CONTROL PREVIO } & Total \\
\cline { 2 - 5 } & NO (tiro directo) & SI & NO \\
\hline \multirow{2}{*}{ Alto } & Recuento & 76 & 149 & 225 \\
\cline { 2 - 5 } & Residuos corregidos & $-2,8$ & 2,8 & \\
\hline \multirow{3}{*}{ Medio } & Recuento & 189 & 258 & 447 \\
\cline { 2 - 5 } & Residuos corregidos &, 0 &, 0 & \\
\hline \multirow{2}{*}{ Bajo } & Recuento & 357 & 450 & 807 \\
\cline { 2 - 6 } & Residuos corregidos & 1,7 & $-1,7$ & \\
\hline \multirow{2}{*}{ Nulo } & Recuento & 11 & 10 & 21 \\
\cline { 2 - 6 } & Residuos corregidos & 1,0 & $-1,0$ & \\
\hline \multirow{2}{*}{ Total } & Recuento & 633 & 867 & 1500 \\
\hline
\end{tabular}

\section{Discusión}

El objetivo de este estudio fue determinar los indicadores de rendimiento ofensivos del floorball en competición de alto nivel. Son escasos los trabajos relacionados con el análisis de la acción de juego en este deporte que nos permitan realizar una discusión comparativa con los resultados obtenidos en este estudio (Prieto y Pérez, 2009; Prieto y Pérez, 2011). En el presente estudio se toma una muestra más amplia que incluye más países y ofrece resultados que pueden ayudar a caracterizar el rendimiento ofensivo de equipos de alto nivel. Destaca, como parámetro general ofensivo, que tan solo el 38,5\% de los lanzamientos que se efectúan llegan a portería en este nivel de competición (20,6 lanzamientos de media por equipo y partido). Esto indica, por un lado, un alto porcentaje de intervención defensiva (35,5\% de tiros tapados), pero por otro, que más de un cuarto de los lanzamientos son errados, lo que muestra la dificultad técnica debido principalmente a la velocidad de ejecución solicitada en la competición en floorball y a las características específicas del material tan ligero utilizado, tanto los palos de material plástico o fibra de vidrio, como en especial de la bola de solo $23 \mathrm{~g}$. En este sentido, hay que recordar que la duración media de las $\mathrm{AO}$ es menor de 9 segundos, resultados similares a los evaluados en fútbol sala (Barbero, 2003), deporte de las mismas dimensiones de campo que el floorball. En el citado estudio, la duración media de las AO es de 8,9 segundos y el $76 \%$ de éstas se realizan por debajo de los 10 segundos $(8,9 \pm 9$ de media y el $57 \%$ en menos de 10 segundos en floorball en el presente estudio). Hemos de precisar, llegados este punto, que ese estudio tiene en cuenta todas las AO, no solo las que acaban en lanzamiento, como es nuestro caso. Estos datos de duración de la AO nos ofrecen indicios para entender los tiempos de participación y de pausa, aspecto tan decisivo en la preparación física y estratégica para que el rendimiento en pista sea óptimo (Hernández, 1996). 
Parece necesario, pues, realizar en el floorball estudios de valoración específica del esfuerzo en competición para poder aplicarlo a los entrenamientos, tal y como se ha hecho en un deporte tan próximo como el hockey patines (Blanco, Enseñat, y Balagüé, 1994; Yagüe, 2005). En este sentido, Yagüe y Pulkkinen (2008) realizan una aproximación a una valoración funcional específica del floorball, con jugadores de la selección española y de un equipo profesional finlandés. Sin duda, las sustituciones ilimitadas en el juego, la estrategia de los entrenadores (que pueden utilizar indistintamente dos o tres líneas de jugadores distintas) y las posibles expulsiones (cerca del $16 \%$ de las AO se realizan en desigualdad numérica), no facilitan esta valoración. Lo que sí se muestra, como en la mayoría de los deportes colectivos y aún más en los que tienen espacios reducidos como el floorball, es que la capacidad de aceleración sobre distancias cortas, en tanto nos permite llegar al móvil antes que el rival (Vales y Areces, 2002), es vital para la obtención de la ventaja y nos marca la importancia de mejorar mediante el entrenamiento esta capacidad.

En cuanto al portero, éste rechaza o para más del $80 \%$ de los lanzamientos que llegan a portería, siendo estos resultados similares (76\%) a los encontrados por Prieto y Pérez (2011) en un estudio en el que la muestra utilizada era de equipos de la liga profesional sueca. La actuación del portero se muestra determinante para el rendimiento de los equipos, indicando una línea prioritaria a seguir en los entrenamientos. Por otra parte, parece importante entrenar los ataques rápidos y con recuperación directa, básicamente en contraataque o búsqueda de segundas oportunidades de lanzamiento, debido a rechaces del portero. Si bien en el floorball no se alcanzan los niveles de eficacia del balonmano, donde se llega a un 90\% de goles en contraataque (Salvador y Mayela, 2009), o el fútbol sala con un 41\% (Álvarez, Puente, Manero y Manonelles, 2004), sí se eleva el nivel medio con respecto al gol de 7,4 \% al 11\%. Además, en el contraataque las AO de éxito alcanzan un $48 \%$, lo que supone obtener menos tiros tapados y fallados, y sobre todo más rechaces del portero, lo que propicia más AO generalmente en zonas próximas a la portería.

En este sentido, la zona más cercana a portería (5C) es determinante en el rendimiento final de los equipos, ya que se consiguen desde ella más del 50\% de los goles; como en el hockey hierba, donde las zonas más próximas a portería obtienen el 72\% de los goles (Piñeiro, 2008). En floorball, y como ha quedado demostrado en este estudio, aquellos equipos que consiguen finalizar en esa zona más AO de las esperadas estadísticamente obtienen mejores resultados. En cuanto al tipo de tiro, el que se efectúa sin control previo disminuye el grado de oposición al tiro y se alcanzan niveles de éxito similares al fútbol, donde el $62 \%$ de los goles se consiguen al primer toque (Molina, 2003), y también en hockey hierba donde se llega al 57\% de eficacia (Piñeiro, 2008). En nuestro estudio el 59,5\% de los goles se han conseguido con el tiro directo. Sin duda, aumentar el número de los lanzamientos de este tipo incrementaría la eficacia, ya que tan solo el $42 \%$ son de este tipo.

En relación a la técnica de lanzamiento, Karlsson (2011) realizó un estudio biomecánico del stick en el slapshot en floorball, lo que en nuestro estudio se denomina lanzamiento con elevación de la pala por encima de la rodilla. Este tipo de estudio tiene influencia sobre el desarrollo del material que influye a su vez en la evolución de la técnica. Muukkonen y Liljelund (2007) y Ragnarsson y Olsson (2002) aportan su visión didáctica del aprendizaje de la técnica. Sin embargo, en base a los resultados aportados en el presente estudio, se puede concluir que la técnica de elevación de la pala por debajo de la rodilla es la más eficiente. 


\section{Conclusión}

El floorball es un deporte de equipo muy rápido, en el que las $\mathrm{AO}$ son de corta duración y donde el contraataque, la recuperación directa y la técnica de lanzamiento mediante la elevación de la pala por debajo de la rodilla son las variables que permiten obtener más posibilidades de éxito en el ataque. Además, la actuación del portero es determinante, ya que rechaza o para más del $80 \%$ de los lanzamientos que llegan a portería. A partir de los resultados obtenidos seguidamente se proponen recomendaciones para el entrenamiento que, esperamos, vengan a redundar en un mayor conocimiento de este deporte y, por extensión, de los indicadores que determinan el rendimiento en ataque.

\section{Aplicaciones prácticas}

Según estos resultados, e intentando sugerir propuestas para la fundamentación del entrenamiento en este deporte a partir de los hallazgos obtenidos en este estudio, se propone aplicar en los entrenamientos actividades concretas para el puesto específico del portero que mejoren su rendimiento y como consecuencia directa, el del equipo. Para hacer descender el grado de oposición al tiro y rebajar el nivel defensivo, hay que dar protagonismo en las sesiones de entrenamiento al tiro directo y a los ataques rápidos (menos de 9 segundos), con 1 a 3 jugadores implicados mediante situaciones de uno contra el portero y ejercicios de rápida ejecución por parejas o por tríos, tratando de finalizar en cualquier caso en la zona frontal a la portería que es la que más éxito obtiene. En este sentido, se sugiere insistir en evitar los lanzamientos, en los entrenamientos y en la competición, desde zonas que han mostrado un nivel significativo de fallo (zonas 4) o priorizar en las zonas de éxito (zonas 5). En las recuperaciones indirectas los resultados nos marcan que hay mucho margen de mejora: se propone utilizar más tiempo a entrenar en este tipo de jugadas de estrategia, procurando generar situaciones sencillas, con varias opciones dependiendo de las características de los jugadores que haya en el equipo, que eviten el contraataque y que finalicen siempre en lanzamiento. En cuanto a la técnica de lanzamiento, es conveniente que el jugador entrene todas las técnicas posibles y adquiera recursos y versatilidad que le permitan superar al adversario y ser más eficaz, pero según los resultados, la técnica más exitosa y, por tanto, la que más debe utilizar, es la elevación de la pala por debajo de la rodilla.

\section{Referencias}

Alonso, J.I., y Argado, F. (2008). Indicadores de rendimiento del saque en frontenis olímpico femenino. RICYDE. Revista Internacional de Ciencias del Deporte. 10(4), 5976.

Álvarez, J.; Puente, J.; Manero, J., y Manonelles, P. (2004). Análisis de las acciones ofensivas que acaban en gol de la liga profesional de fútbol sala española. RED. Revista de Entrenamiento Deportivo, Tomo 18, No4, 27-32.

Anguera, M.T. (2009). Los deportes de equipo estudiados desde la metodología observacional: ¿diferentes perspectivas de la misma realidad? En actas publicadas del II Congreso Internacional de Deportes de Equipo (CD). Alicante: Editorial Alto Rendimiento.

Barbero, J.C. (2003). Análisis cuantitativo de la dimensión temporal durante la competición de fútbol sala. Revista Motricidad. European of Journal Movement. 10, 143-163.

Blanco, A.; Enseñat, A., y Balagüé, N. (1994). Hockey sobre patines: niveles de frecuencia cardíaca y lactacidemia en competición y entrenamiento. Apunts,36, 2636. 
Gabbett, T. J. (2010). GPS Analysis of Elite Women's Field Hockey Training and Competition. Journal of Strength and Conditioning Research, 24, 1321-1324.

Geithner, C.A.; Lee, A. M., \& Bracko, M. R. (2006). Physical and performance differences among forward defense men and goalies in elite women's ice hockey. Journal of Strength and Conditioning Research, 20, 500-505.

Gómez, M. A., \& Pollard, R. (2011). Reduced home advantage for basketball teams from capital cities in Europe. European Journal of Sport Science, 11, 143-148.

Gómez, M. Á., y Lorenzo, A. (2005). Diferencias entre equipos ganadores y perdedores en el rendimiento de competición en baloncesto femenino. Kronos: la Revista Científica de Actividad Física y del Deporte, no 8, julio/diciembre, 16-22.

Gómez, M.Á.; Lorenzo, A.; Ortega, E., y Olmedilla, A. (2007). Diferencias de los indicadores de rendimiento en baloncesto femenino entre ganadores y perdedores en función de jugar como local o como visitante. Revista de Psicología del Deporte, 16(1), 41-54.

Hernández, J. (1996). Tiempo de participación y de pausa y de las incidencias reglamentarias en los deportes de equipo (I). RED. Revista de Entrenamiento Deportivo, Tomo X, 1 y 2, 23-30.

Hugues, M., \& Bartlett, R (2002). The use of performance indicators in performance analisys. Journal of Sports Sciences, 20, 739-754.

Karlsson, J. (2011). Analysis of floorball sticks using high speed camera and Abaqus. An explicit simulation of the dynamic behaviour during a slapshot. Master's Thesis in the International Master's Programme Applied Mechanics. Chalmers University of Technology, Göteborg.

Kingman, J., \& Dyson, R. (2001). The scientific literature available on roller hockey. Journal of Human Movement Studies. 41, 415-433.

Lago, C. \& Martín, R. (2007). Determinants of possession of the ball in soccer. Journal of Sport Sciences, 25, 969-974.

Lago, C. (2009). The influence of match location, quality of opposition, and match status on possession strategies in professional relation football. Journal of Sports Sciences, 27, 1463-1469.

Laird, P., \& Sutherland, P. (2003). Penalty corners in field-hockey: a guide to success. International Journal of Performance Analysis in Sport, 3, 9-26.

Manzano, A.; Lorenzo, A., y Pacheco, J. L. (2005). Análisis de la relación entre el tipo de defensa y la ejecución de bloqueos directos y el éxito en la finalización de las posesiones en baloncesto. Kronos: la Revista Científica de Actividad Física y del Deporte, 8, 62-70.

Molina, J. (2003). Estudio de la lateralidad del jugador en la consecución de los goles a primer toque en la Liga Española de 10 División en la temporada 2000/01. El Entrenador Español, 90, 34-44.

Muukkonen, M., \& Liljelund, J. (2007). IFF Floorball Development Programme. Canada International Semminary.

Ortega, E.; Fernández, R.; Ubal. M.; Lorenzo, A., y Sampaio, J. (2010). Indicadores de rendimiento defensivo en baloncesto en los ganadores y perdedores. RICYDE. Revista Internacional de Ciencias del Deporte. 19(6), 100-111.

Paavilainen, A. (2007). Team tactics. Teaching team tactics in floorball; instructions and drills. Suecia: International Floorball Federation.

Pardo, A., y Ruíz, M.A. (2005). Análisis de datos con SPSS 13 Base. Madrid: McGrawHill. 
Piñeiro, R (2008). Observación y análisis de la acción de gol en hockey hierba. Sevilla: Wanceulen.

Pollard, R., \& Gómez. M.A. (2007). Home advantage analysis in different basketball leagues according to team ability. Iberian congress on basketball research, 4, 61-64.

Prieto, M., y Pérez, J. (2009). Análisis del rendimiento en el floorball de máximo nivel (I): validación experta de una herramienta de análisis de las acciones ofensivas que acaban en lanzamiento. II Congreso Internacional de Deportes de Equipo. Universidade da Coruña.

Prieto, M., y Pérez, J. (2010). El rendimiento ofensivo en el floorball de competición: determinación de las variables de estudio. RED. Revista de Entrenamiento Deportivo. Tomo XXIV. No 2, 13-20.

Prieto, M., y Pérez, J. (2011). Análisis del rendimiento ofensivo en la liga sueca de floorball: resultados preliminares. Kronos: la Revista Científica de Actividad Física y Deporte, Vol. X (1), 89-96.

Ragnarsson, T., \& Olsson, Ch. (2002). Unihockey para todos. IFF. Jornadas Internacionales de Unihockey - Floorball. Instituto Andaluz del Deporte. Fuengirola.

Salvador, J., y Mayela, F. (2009). Análisis descriptivo del balonmano. Tiempos de juego, tipos de pausa y efectividad del contraataque. Lecturas: Educación Física y Deportes. Año 14, no 138. Recuperado 13 de octubre, 2010. Disponible en http://www.ef.deportes.com/efd138.

Sampaio, J.; Drinkwater, E. J., \& Leite, N. (2010). Effects of season period, team quality, and playing time on basketball players' game-related statistics. European Journal of Sport Sciences, 10, 141-149.

Sampaio, J.; Ibáñez, S. J.; Gómez, M. A.; Lorenzo, A., \& Ortega, E. (2008). Game location influences basketball players' performance across playing positions. International Journal of Sports Psychology, 39(3), 205-216.

Sampaio, J.; Lago, C.; Casais, L., \& Leite, N. (2010). Effects of starting score-line, game location and quality of opposition in basketball quarter score. European Journal of Sport Sciences, 10, 391-396.

Thomas, J., y Nelson, J. (2007). Métodos de investigación en actividad física. Badalona: Paidotribo.

Vales, A., y Areces, A. (2002). Aproximación conceptual a la velocidad en deportes de equipo. El caso del fútbol. Apunts: Educación Física y Deporte, 69, 44-58.

Vales, A., y Areces, A. (2009). Planificación del entrenamiento en los deportes de equipo: bases conceptuales y estado de la cuestión. RED. Revista de Entrenamiento Deportivo, Tomo XXIII. No 1, 5-14.

Yagüe, P. (2007). Hockey sobre patines: estudio de las demandas fisiológicas en competición, análisis del perfil fisiológico funcional, desarrollo y validación de un modelo de valoración funcional específica orientado al jugador de campo. Tesis doctoral publicada, servicio de publicaciones, Universidad de Oviedo.

Yagüe, P., y Pulkkinen, S. (2008). Propuesta para la valoración funcional en el floorball de competición. Lecturas: Educación Física y Deportes. Año $13-N^{\circ} 126$. Recuperado 12 de diciembre, 2012, disponible en http://www.efdeportes.com/ efd126. 